\title{
Estimation of Maize Planting Area through the Fusion of Multi-source Images"
}

\author{
Xiaohe Gu, Yuchun Pan ${ }^{* *}$, Xin He, and Jihua Wang \\ Beijing Agriculture Information Technology Research Center, Beijing 100097, China \\ \{guxh, panic\} anercita.org.cn
}

\begin{abstract}
The limitations on spatial resolution and on the availability and measurement accuracy of remote sensing images are the primary problems in the estimation of the large-scale planting area for maize. The integration of mid- and low-resolution images is the one of primary methods used for the estimation of large-scale crop planting areas using remote sensing. The use of a single-temporal thematic mapper (TM) image results in a low accuracy of maize recognition, so a mid-scale time-series normalized difference vegetation index (NDVI) dataset, which was derived from the fusion of the moderate-resolution imaging spectroradiometer (MODIS) and TM images based on the wavelet transform, was established. The planting area was estimated using the minimum distance model and the accuracy was evaluated using in-situ samples. The results show that the estimation of the maize-sown area based on the time-series NDVI information of the integrated images reached high levels of gross and position accuracy ( $89 \%$ and $90 \%$ ), indicating that this method could fully utilize the time-series information from the MODIS images and the spatial resolution of a TM image. The use of the difference in phenophases among fall crops enables the effective classification of the spatial distribution of these crops.
\end{abstract}

Keywords: maize, planting area, MODIS, TM, remote sensing, wavelet transform, minimum distance.

\section{Introduction}

Maize is one of the primary crops in China, the planting area and yield of which comprise one-fifth of all crops [1]. Accurate information on the planting area and distribution of maize are important for effective yield estimation, agricultural management, and national food security [2]. The spatial characteristics of maize distribution in China include complex planting structures and fragmented planting parcels. The limitations on spatial resolution and on the availability and measurement of the accuracy of remote sensing images are the primary problems in the estimation of the large-scale planting area for maize [3].

\footnotetext{
* The National Natural Science Funds (No. 41001199), Beijing Excellent Talent Program (No. PYZZ090416001998) and Beijing Scientific New Star Program (No. 2010B024).

** Corresponding author.
} 
Previous studies show that the integration of mid- and low-resolution images is the primary methods used for investigating large-scale crop planting areas [4-7]. A mid-resolution image has a high level of accuracy in crop recognition, but lacks availability and coverage [8 - 9]. On the other hand, a low-resolution image enables the acquisition of long time-series information that match crop growth [10 - 11]. Thus, utilizing mid- and low-resolution images to conduct crop recognition through the integration of the multi-source data is necessary. Currently available studies on crop planting area estimation primarily focus on winter wheat [12-13] and rarely emphasize maize. A number of crops are synchronized with maize, so this crop is of great significance for research on the estimation of planting areas through the integration of mid- and low-resolution images.

The Yuanyang County in Henan province was selected as the study area. The data used in the current paper included multi-temporal moderate resolution imaging spectroradiometer (MODIS) normalized difference vegetation index (NDVI) images and a single-temporal thematic mapper (TM) NDVI image. A mid-scale time-series NDVI dataset, which was derived from the fusion of the MODIS and TM images based on wavelet transform, was established because of the low accuracy of maize recognition when using only a single-temporal TM image. The planting area was then estimated using the minimum distance model and the accuracy was evaluated using in-situ samples.

\section{$2 \quad$ Study Area and Data Preprocess}

\subsection{Study Area}

The Yuanyang County in Henan province, a typical agricultural region where the planting structures are stable and the fall crops include maize and paddy, was chosen as the study domain. The phenological differences of the crops are often used to select the temporal remote sensing data for crop recognition. Based on the crop phenology in Yuanyang County, growing time is generally from mid-June to mid-October. The synchronized fall crops include paddy, peanut, and soybean, which tend to obstruct maize recognition.

\subsection{Data Preprocess}

The remote sensing data used in the current paper included MODIS and TM images covering the entirety of Yuanyang County. The Earth Observing System/MODIS data were composed of the NDVI images with a maximum synthesis of $16 \mathrm{~d}$ downloaded from the NASA website. The MODIS images were captured from May 24 to October 30,2008 , with a spatial resolution of $250 \mathrm{~m}$. On the other hand, the TM image was captured on August 25, 2008, with a spatial resolution of $30 \mathrm{~m}$. The data preprocess included geometric correction, atmospheric calibration, and projection transformation. The polynomial model of bilinear interpolation, which could ensure an error of less than 0.5 pixels, was used for the geometric correction. The $6 \mathrm{~S}$ model was used for the atmospheric calibration. The projection type used for all spatial data used the Universal Transverse Mercatol system, whereas the geographic coordinate system was WGS 84 . 

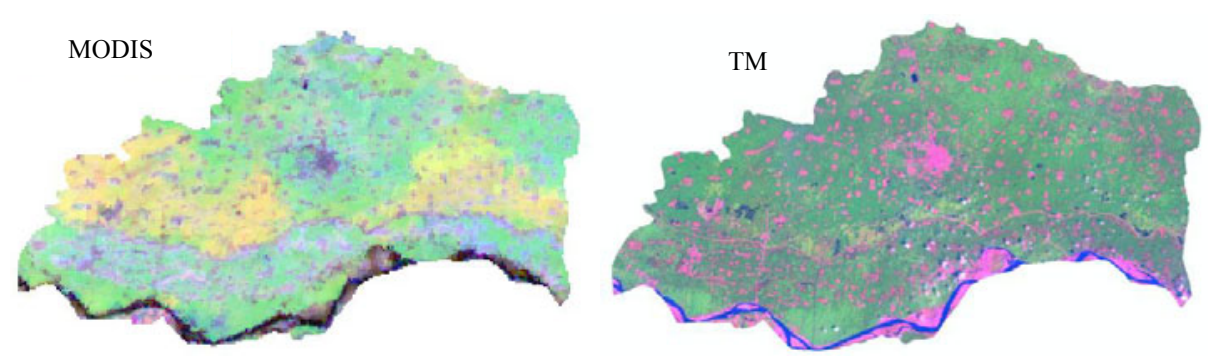

Fig. 1. MODIS and TM images covering Yuanyang County in 2008

Farmland parcel data, which were digitized from SPOT5 fusion images, provided the basic unit for the in-situ sample location and accuracy evaluation. The in-situ data included 49 sample parcels from August 2008, the attribute of which includes the parcel and planting area percentages for maize. The in-situ samples were primarily used to evaluate the accuracy of the method.

\section{$3 \quad$ Method and Technical Process}

The technical processes for the estimation of the maize planting area using integrated MODIS and TM images including wavelet transform fusion, reconstruction of time-series NDVI, classification by minimum distance model, and accuracy evaluation, are discussed in this section.

\subsection{Fusion Model of Wavelet Transform}

According to the theory and algorithm of wavelet transform, an image could be divided into one approximate component and three detail components. The approximate and detail components represent low- and high-frequency information, respectively. Using the inverse wavelet transform, high frequency information derived from the high-resolution image and low-frequency information derived from the multi-spectrum image could be transformed into the fusion image.

The algorithm of the wavelet transform model derived from the orthogonal basis of compact supported wavelets can be shown as follows:

$$
\left\{\begin{array}{l}
C_{j+1}=H_{r} H_{c} C_{j} \\
D_{j+1}^{1}=H_{r} G_{c} C_{j} \\
D_{j+1}^{2}=G_{r} H_{c} C_{j} \\
D_{j+1}^{3}=G_{r} G_{c} C_{j}
\end{array}\right.
$$


where $\mathrm{J}$ is the decomposing order of the wavelet transform, $\mathrm{C}$ is the matrix of the original image, $\mathrm{G}$ is the high-pass filter, $\mathrm{H}$ is the low-pass filter, $\mathrm{C}_{\mathrm{j}}$ is the low-frequency component, and $D_{j}$ represents the three high-frequency components.

The key parameters of the wavelet transform fusion model include the wavelet basis, decomposition level, and fusion rule. The wavelet fusion is unable to provide a uniform theory and method for multi-source sensors because of the various remote sensor types and different spatial resolutions. Thus, performing numerous experiments was necessary to analyze the optimal wavelet basis, decomposition level, and fusion rule of the wavelet fusion between the MODIS and TM images.

After several experiments, the fusion of the wavelet transform between the MODIS and TM data was conducted using the BIOR6.8 wavelet basis and three decomposition layers and employing the fusion rule of high-low frequency conversion. Figures 2 and 3 show the final fusion image.

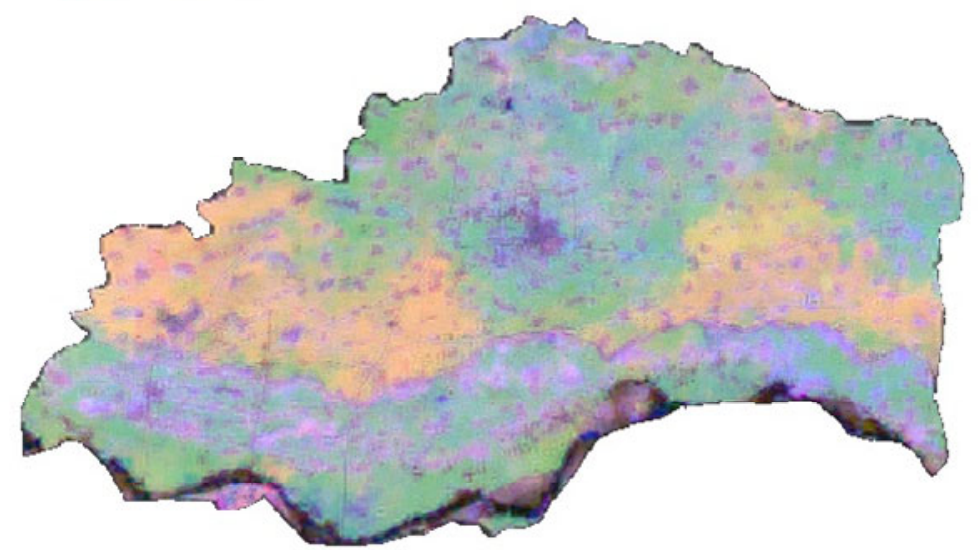

Fig. 2. Fusion image from the MODIS and TM images based on the wavelet transform

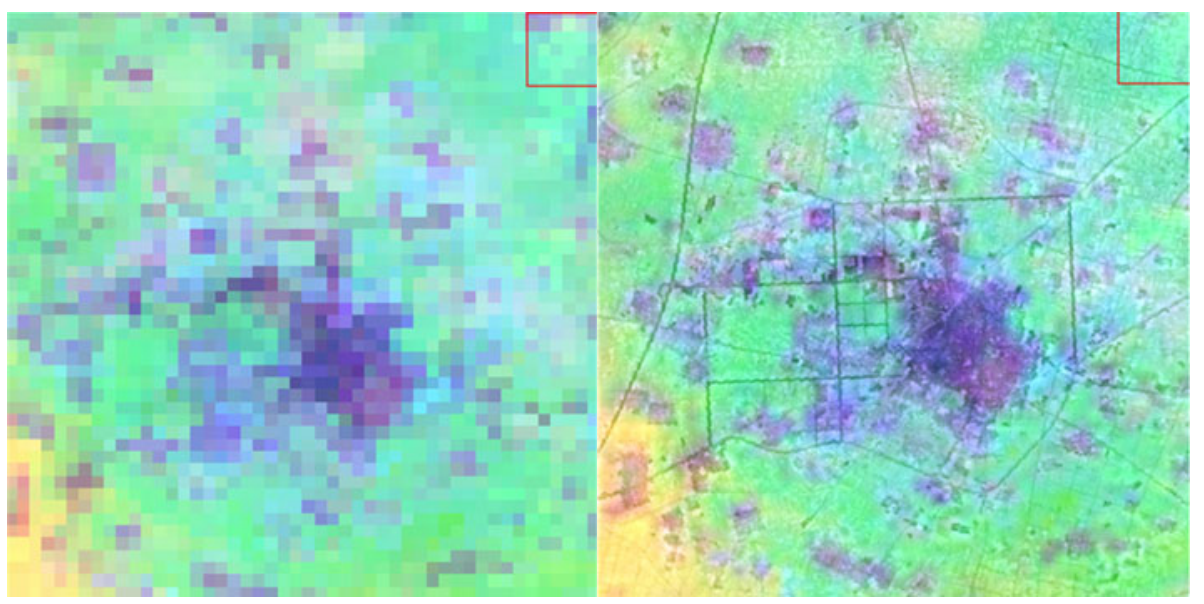

a) Original images of MODIS

b) Final fusion images

Fig. 3. Contrast between portions of the original MODIS images and the final fusion image 


\subsection{Reconstruction and Analysis of the Time-Series NDVI}

The NDVI is the optimal indicator for the features, growth condition, and coverage of vegetation. In the current study, the standard growth curves of the primary fall crops were obtained using the time-series fusion image from the NDVI with a resolution of $30 \mathrm{~m}$. First, using the located in-situ samples, pure parcel samples from the NDVI time-series fusion images, including maize, paddy, peanut, and soybean, were selected.

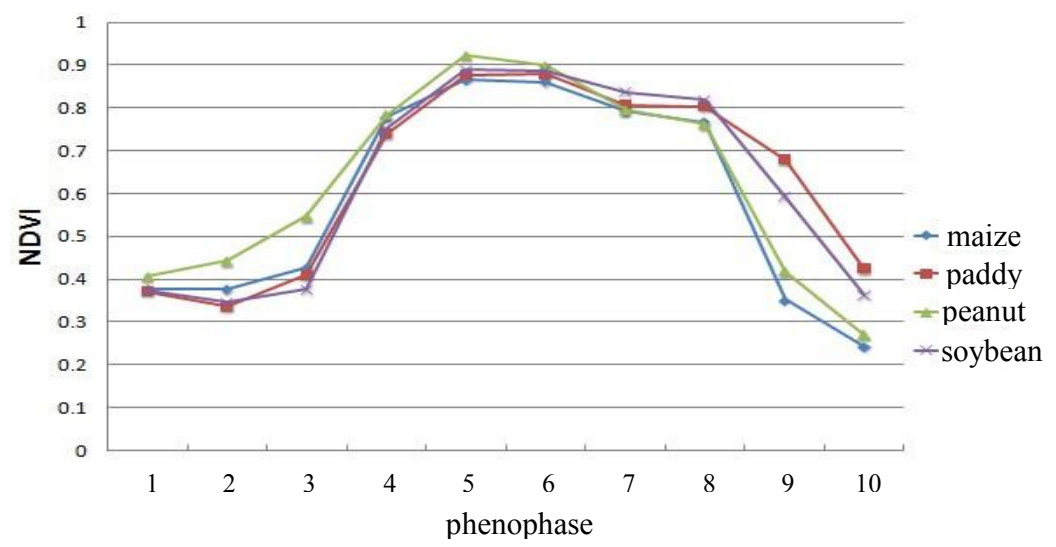

Fig. 4. NDVI time-series curves of the fall crops

As shown in Figure 4, the reflectance of the red band decreased when the coverage and leaf area index of the four crops increased, whereas the reflectance of the near infrared band increased along with the phenological changes. Thus, each of the four crops had peaks in the NDVI time-series curves.

The peak of maize in the NDVI curve appeared between the end of July and the first week of August, which corresponded to the grain-filling stage. The peak resulted from the end of the nutrition growth and the beginning of the complete reproductive growth of maize.

The features of the NDVI curves of the four crops were different to a certain extent. At the early-growth stages, the NDVI value of maize was difficult to distinguish because it was similar to that of paddy and soybean. However, at the late-growth stages, the NDVI values of maize and peanut rapidly decreased and showed significant differences with the NDVI values of paddy and soybean.

\subsection{Minimum Distance Classifier}

The selection of an effective distance measurement is important for the algorithm of the minimum distance classifier. The universal distance parameters include the Euclidean distance (ED) and the Mahalanobis distance, among others. In the current study, the typical ED was selected to determine the pixel classification using the minimum distance model. The function of Euclidean distance is as follows: 


$$
\mathrm{d}_{\mathrm{E}}=\sqrt{\left(\mathrm{NDVI}_{\mathrm{r}}-\mathrm{NDVI}_{\mathrm{t}}\right)^{2}}
$$

\subsection{Accuracy Evaluation}

The in-situ sample parcels were assumed to have the real values, and the accuracy of the estimation of the maize planting area was evaluated based on the fusion of the MODIS and TM images with the indexes of position accuracy and gross accuracy.

The contributions of the different parcels varied because of the area differences of each farmland parcel. Taking the parcel area as weight, the function of the position accuracy is as follows:

$$
\mathrm{K}_{\mathrm{i}}=\frac{\sum_{\mathrm{i}=1}^{\mathrm{n}}\left(1-\left|\mathrm{p}_{\mathrm{i}}-\mathrm{p}_{\mathrm{io}}\right|\right) * \mathrm{~A}_{\mathrm{i}}}{\sum_{\mathrm{i}=1}^{\mathrm{n}} \mathrm{A}_{\mathrm{i}}} * 100 \%
$$

where $P_{i}$ is the estimated percentage of maize in one parcel, $P_{i o}$ is the maize percentage of the in-situ sample parcel, $A_{i}$ is the area of a certain parcel and $n$ is the number of in-situ samples.

The evaluation of the gross accuracy was performed using the planting area account of all in-situ sample parcels as the true value. The function of the gross accuracy is as follows:

$$
\mathrm{K}_{\omega}=\left(1-\frac{\left|\sum_{\mathrm{i}=1}^{\mathrm{n}} \mathrm{p}_{\mathrm{i}} * \mathrm{~A}_{\mathrm{i}}-\mathrm{A}_{\mathrm{o}}\right|}{\mathrm{A}_{\mathrm{o}}}\right) * 100 \%
$$

where $\mathrm{P}_{\mathrm{i}}$ is the estimated percentage of maize in one parcel, $\mathrm{A}_{\mathrm{i}}$ is the area of a certain parcel, $n$ is the number of in-situ samples of maize and $A_{o}$ is the account of maize planting area of the in-situ sample parcels.

\section{$4 \quad$ Results}

The time-series curves of the four crops show that the NDVI features of several crops were similar to that of maize. At the seedling stage, the NDVI values of maize, paddy, and soybean were near that of one another, indicating that the growth conditions of the three crops were similar. At the jointing stage, the NDVI value of maize was close to that of the other crops, which were hardly distinguished. At the maturity stage, the NDVI value of maize was close to that of peanut when they were divided. However, the NDVI value of peanut was different with that of maize at the seedling stage, so the spatial distribution could be extracted using the stratified classification method.

Considering the differences in terms of phenomena and growing process among the studies crops, the spatial distribution of the NDVI values of peanut and maize at the seedling stage were extracted first. Then, the total spatial distributions of maize and peanut were distinguished based on their NDVI characteristics at the maturity stage. Finally, the joint of both spatial distributions was considered as that of maize, through which the account of the maize planting area in Yuanyang County could be estimated. 
The maize planting area was also derived using a supervised classification of the in-situ samples. Considering the in-situ sample parcels as the true value, the accuracy of the supervised classification and time-series analyses were evaluated. The position and gross accuracies of maize derived using supervised classification were $62.89 \%$ and $49.01 \%$, respectively. The estimated accuracies were derived from an improved time-series analysis from which the position and gross accuracies were $78.76 \%$ and $88.57 \%$, respectively.

Table 1. Analysis of the accuracy of the maize planting area derived from the supervised classification and time-series analysis methods

\begin{tabular}{ccc}
\hline method & position accuracy & gross accuracy \\
\hline supervised classification & $62.89 \%$ & $49.01 \%$ \\
time-series analysis & $78.76 \%$ & $88.57 \%$ \\
\hline
\end{tabular}

\section{Conclusion and Discussion}

The fusion of MODIS and TM images based on wavelet transform enabled the plotting of the standard growth curves of the primary fall crops with a $30 \mathrm{~m}$ resolution, and the maize planting area was estimated using a minimum distance classifier. The results show that the estimation of the maize-sown area based on the time-series NDIV information of the fusion images could yield high values of gross and position accuracy (89\% and $90 \%$ ), indicating that this method could fully utilize the time-series information from the MODIS images and the spatial resolution of a TM image.

The present study requires further improvement. First, studying the estimation method for the maize planting area using daily time-series images is necessary because the MODIS NDVI data are synthesized from the maximum synthesis value of $16 \mathrm{~d}$ and because the phenomena observed from the fall crops are similar. Second, although the current study improved the spatial resolution of time-series data from 250 to $30 \mathrm{~m}$, the spatial character of maize distribution, including the complex planting structure and fragmented planting parcels, result in mixed pixels. Therefore, studying soft classification or mixed pixel decomposition is necessary to reduce the effect of mixed pixels.

\section{References}

[1] Li, Y., Tan, K.: A preliminary study on the method for corn yield estimation by remote sensing in north China. Quarterly Journal of Applied Meteorology 6(supplement), 33-41 (1995)

[2] Chen, S., Liu, Q., Chen, L., et al.: Review of research advances in remote sensing monitoring of grain crop area. Transactions of the Chinese Society of Agricultural Engineering 21(6), 166-171 (2005)

[3] Gu, X., Pan, Y., Zhu, X., et al.: Consistency study between MODIS and TM on winter wheat plant area monitoring. Journal of Remote Sensing 11(3), 350-358 (2007) 
[4] Van Niel, T.G., McVicar, T.R.: A simple method to improve field-level rice identification: toward operational monitoring with satellite remote sensing. Australian Journal of Experimental Agriculture 43, 379-387 (2003)

[5] Xiao, X., Stephen, B., et al.: Mapping paddy rice agriculture in South and Southeast Asia using multi-temporal MODIS images. Remote Sensing of Environment 100, 95-113 (2006)

[6] Gao, J.F., Pan, G.X., Jiang, X.S., et al.: Land-use induced changes in topsoil organic carbon stock of paddy fields using MODIS and TM/ETM analysis: A case study of Wujiang County, China. Journal of Environmental Sciences 20, 852-858 (2008)

[7] Wu, M., Wang, C., Niu, Z.: Mapping paddy fields in large areas, based on time series multi-sensors data. Transactions of the CSAE 26(7), 240-244 (2010)

[8] Zhang, J., Shen, K., Pan, Y., et al.: HJ-1 remotely sensed data and sampling method for wheat area estimation. Scientia Agricultura Sinica 43(16), 3306-3315 (2010)

[9] Ma, L., Gu, X., Xu, X., et al.: Remote sensing measurement of corn planting area based on field-data. Transactions of the CSAE 25(8), 147-151 (2009)

[10] Yang, X., Zhang, X., Jiang, D.: Extraction of multi-crop planting areas from MODIS data. Resources Science 26(6), 17-22 (2004)

[11] Xu, W., Zhang, G., Fan, J., et al.: Remote sensing monitoring of winter wheat areas using MODIS data. Transactions of the CSAE 23(12), 144-149 (2007)

[12] Feng, M., Yang, W., Zhang, D., et al.: Monitoring planting area and growth situation of irrigation- land and dry-land winter wheat based on TM and MODIS data. Transactions of the CSAE 25(3), 103-109 (2009)

[13] Gai, Y., Li, X., Li, X., et al.: Agricultural drought monitoring based on TM and MODIS remote sensing data - a case study of HebeiProvince. Journal of Nature Disasters 17(6), 91-95 (2008)

[14] Wang, H., Zhou, J., Wu, W.: Applications of wavelet analysis to remote sensing image processing. Remote Sensing Information 01, 93-99 (2009)

[15] Zhang, A., Li, N.: Research and analysis of multi-sources remotely sensed imagery fusion on the basis of wavelet transform technique. Modern Surveying and Mapping 32(5), 39-41 (2009)

[16] Han, L., Chen, Q., Han, T., et al.: Study on 3S-Based Estimation of Cotton-growing Area. Arid Zone Research 25(2), 207-211 (2008)

[17] Jing, R., Chun, L.: Improved minimum distance classifier — weighted minimum distance classifier. Computer Applications 25(5), 992-994 (2005)

[18] Gu, X., Pan, Y., He, X., et al.: Measurement of sown area of winter wheat based on per-field classification and remote sensing imagery. Journal of Remote Sensing 14(4), 789-805 (2010) 